\title{
Reading of Everyday Texts by People with Aphasia : Do Advance Organisers Help?
}

\author{
Hanh Nguyen ${ }^{12}$, Julie Morris ${ }^{1}$, Janet Webster ${ }^{1}$, and Lyndsey Nickels ${ }^{3}$
}

1. Speech and Language Sciences, School of Education, Communication and Language Sciences, Newcastle University, Newcastle, UK

2. International Doctorate for Experimental Approaches to Language and Brain (IDEALAB), Potsdam University, Germany, Groningen University, Netherlands, Trento University, Italy, Newcastle University, UK, and Macquarie University, Australia.

3. ARC Centre of Excellence in Cognition and its Disorders, Department of Cognitive Sciences, Macquarie University, Sydney, Australia

Correspondence to Hanh Nguyen, Email: t.nguyen1@newcastle.ac.uk

\section{Background}

Many people with aphasia (PWA) experience difficulty reading and report a preference for texts accompanied with pictures (Knollman-Porter, Wallace, Hux, Brown, \& Long, 2015). Pictures, a form of advance organisers, have been shown to facilitate text processing and comprehension in unimpaired readers (Glenberg \& Langston, 1992). There is also evidence that reading accuracy improves in PWA when texts are presented with pictures (Dietz, Hux, McKelvey, Beukelman, \& Weissling, 2009; Dietz, Knollman-Porter, \& Hux, 2014). However, previous studies did not use naturalistic (i.e. everyday texts) but highly-constrained texts (i.e. texts created by researchers based on pictures selected for the experiments). In addition, an important aspect of the reading process, reading speed, has not been examined.

It has also been found that, similar to unimpaired readers, PWA show greater difficulty in comprehending details and implied ideas in texts compared to main ideas and stated ideas (Webster, Morris, Howard, \& Garraffa, 2018). It is, however, unclear whether advance organisers can benefit PWA regarding the understanding of a particular type of textual information.

\section{Aims}

The purpose of this study was to investigate the extent to which advance pictorial organisers influence PWA's reading speed and accuracy for everyday texts.

\section{Methods \& Procedures}

Thirty healthy people and eleven PWA participated in the study. The healthy control participants were aged 50 to 85 and had no significant cognitive deficits as suggested via cognitive screening. Participants with aphasia were aged 64 to 83 and varied in their aphasia severity and reading ability. Participants read 45 news articles which were originally selected from an online news website and were 110-150 words long. Each article was preceded by either a related picture, an unrelated picture, or no picture and was followed by four multiple-choice questions. The questions assessed the understanding of four types of textual information: stated main ideas, implied main ideas, stated details, and implied details. Linear and generalized linear mixed-effects analyses were performed to examine the effect of picture conditions on reading times and accuracy, respectively. 


\section{Outcomes \& Results}

A main effect of participant group, $\chi^{2}(1)=33.1, p=3.064 \mathrm{e}-07$, indicated that PWA had significantly longer reading times than the healthy controls. There was also a main effect of picture condition for reading times, $\chi^{2}(2)=7, p=.03$. There was, however, no significant interaction between group and picture condition, $\chi^{2}(2)=.3, p=.87$.

Separate analyses of reading times for each group showed that both groups had significantly shorter reading times in the related condition (control: $M=40.69, S D=17.65$, PWA: $M=75.42$, $S D=30.84$ ) than the no-picture condition (control: $M=41.72, S D=16.98, t(1278)=4.56$, $p<.0001$; PWA: $M=82.44, S D=33.15, t(446)=2.8, p=.015)$. The reading times in the no-picture condition were significantly shorter than in the unrelated condition for controls $(M=42.52$, $S D=16.98, t(1278)=2.86, p=.012)$, but not for participants with aphasia $(M=83.83, S D=32.45$, $t(446)=1.87, p=.15)$. The unrelated and no-picture conditions did not differ from each other in either groups (control: $t(1278)=1.7, p=.206$; PWA: $t(446)=.93, p=.623$ ).

Group had a main effect on accuracy, $\chi^{2}(1)=25.2, p=5.260 \mathrm{e}-07$, indicating that PWA had significantly lower accuracy than the healthy controls. There was no effect of picture condition, $\chi^{2}(2)=1.2, p=.56$, and no significant interaction between group and picture condition, $\chi^{2}(2)=.4, p=.81$, as well as between picture condition and types of textual information, $\chi^{2}(2)=1.6, p=.44$.

\section{Conclusions}

The results indicated that PWA benefit from advance pictorial organisers as do people without aphasia, resulting in reduced reading speed for everyday texts. This suggests that text processing can be supported by top-down processing with readers integrating contextual information provided through advance pictorial organisers. Considering clinical implications, advance provision of pictorial organisers could be used as a therapeutic strategy to support reading by increasing PWA's reading speed.

\section{References}

Dietz, A., Hux, K., McKelvey, M. L., Beukelman, D. R., \& Weissling, K. (2009). Reading comprehension by people with chronic aphasia: A comparison of three levels of visuographic contextual support. Aphasiology, 23(7-8), 1053-1064.

Dietz, A., Knollman-Porter, K., \& Hux, K. (2014). Supported reading comprehension for people with aphasia: Photographic and linguistic supports. Journal of Medical Speech Language Pathology, 21(4), 319-331.

Glenberg, A. M., \& Langston, W. E. (1992). Comprehension of illustrated text: Pictures help to build mental models. Journal of Memory and Language, 31(2), 129-151.

Knollman-Porter, K., Wallace, S. E., Hux, K., Brown, J., \& Long, C. (2015). Reading experiences and use of supports by people with chronic aphasia. Aphasiology, 29(12), 1448-1472. 
Webster, J., Morris, J., Howard, D., \& Garraffa, M. (2018). Reading for meaning: What influences paragraph understanding in aphasia? American Journal of Speech-Language Pathology, 27(1S), 423-437. 